\title{
Can a Nutritional Support Team improve the nutritional standard of care in patients with fractured neck of femur?
}

\author{
N. M. Smith ${ }^{1}$, A. Padwick ${ }^{2}$, N. Pendleton ${ }^{3}$, A. Myers ${ }^{2}$, J. L. Shaffer ${ }^{2}$ and K. Farrer ${ }^{1}$ \\ ${ }^{1}$ Nutrition and Dietetic Department, Salford Primary Care Teaching Trust, Salford, UK, ${ }^{2}$ Intestinal Failure Unit, Salford \\ Royal NHS Foundation Trust, M6 8HD, UK and ${ }^{3}$ Orthopaedic Directorate Salford Royal NHS Foundation Trust, \\ M6 $8 H D, U K$
}

Despite a growing awareness of the dramatic impact on quality of life and treatment costs ${ }^{(1,2)}$ patients with a hip fracture often have a poor nutritional status ${ }^{(3)}$ which is associated with increased risk of complications, morbidity and mortality. Adequate nutrition is essential in the care of the hip fracture patients in order to achieve recovery without complications. The aim of this pilot was to investigate the effects of an improved care intervention in relation to nutritional status. Following patient consent, Mid Arm Circumference (MAC) and Hand Grip Strength were measured on admission and discharge and length of stay recorded (Table 1).

From January 2008 to June 2008, a total of 81 patients over the age of 75 years with a fractured femur were seen by the Nutrition Support Team (NST). Seventy six patients $(94 \%)$ were screened by the NST on admission using the Malnutrition Universal Screening Tool (MUST); $43(57 \%)$ of patients were assessed as medium or high risk. Due to cognitive impairment only 31 and 21 patients respectively consented to MAC and grip strength being measured on admission and discharge,

\begin{tabular}{lccccc}
\hline & \multicolumn{2}{c}{ Low risk MUST on admission } & & \multicolumn{2}{c}{ Medium and high risk MUST on admission } \\
\cline { 2 - 3 } \cline { 5 - 6 } & Admission & Discharge & & Admission & Discharge \\
\hline Median & 28.4 & 28.7 & & 25.6 & $(14-32)$ \\
MAC cm (range) & $(21.5-34)$ & $(22-34)$ & & $(13.5-32)$ & 15.6 \\
Median & 17 & 19 & $(8-30)$ & $(3-21)$ & $(5-23)$ \\
Hand grip kg (range) & $(6-28)$ & $16(4-39)$ & & $22(3-65)$ \\
Median & - & & & \\
Length of stay; days (range) & & & & \\
\hline
\end{tabular}

Sixty five percent of all patients were commenced on nutritional support; only $8 \%$ of patients required artificial nutrition support; $54 \%$ required two oral sip feeds per day; providing an additional $660 \mathrm{kcal}$ and $28 \mathrm{~g}$ protein. Data from 2007 highlighted only $19 \%$ of orthopaedic patients were screened within $24 \mathrm{~h}$ of admission, by introducing a team approach this improved documentation and ensured accuracy. The average length of stay was reduced to 23 days; a reduction of 7 days when compared to 2005/6 data. To conclude, nutritional standards can be improved and length of stay reduced in a high risk patient population by introducing a multi-disciplinary approach to nutrition.

1. Allman RM. (1997) Clin Geriatr Med 13, 421-436.

2. Barczack CA, Barnett RI, Childs EJ et al. (1997) Adv Wound Care 10, 18-26.

3. Bastow MD, Rawling J \& Allison SP (1983) Lancet 1(8317), 143-146. 